\title{
SIMULATION OF THE DYNAMIC TRANSISTOR NEGATRONS
}

\author{
PAVLO MOLCHANOV, ARUN K. MISRA*, HELEN LINNIK \\ and PAVLO MULYAR
}

\author{
Vinnytsia State Technical University, 95 Chmelnitske Shose, \\ Vinnytsia, 286021, Ukraine
}

(Received 24 November 1999; In final form 28 January 2000)

\begin{abstract}
The simulation principles of monolithic microwave dynamic transistor negatrons (circuits with negative differential active resistance) are introduced. The non-linear model has been developed on the basis of non-linear charge model. The equivalent circuit and Volterra series were used for the calculation of dynamic negatrons' parameters. The expressions were obtained, which give the linear relation between current and voltage charges and allow calculating the transferring characteristics. Experimental oscillators are described, which confirm the theoretical predictions.
\end{abstract}

Keywords: Dynamic transistor negatrons; Volterra series; Non-linear model; Experimental oscillators

Depending on the functional principles, the devices with negative differential active resistance, which are called negatrons [1-3], may be classified into three groups:

- negatrons with negative resistance and S-mode static amperevoltage characteristics (dynisters, trinistors);

- negatrons with negative conductivity and N-mode static amperevoltage characteristics (tunnel, Gun diodes);

- dynamic negatrons with negative differential active resistance for alternate current only (avalanche diodes and inverted

\footnotetext{
*Corresponding author. Tel.: (+380) (432) 325 718, Fax: $(+380)(432) 465772$,
} e-mail:mpavlo@mail.vinnica.ua 
common collector transistor circuits) $[3,4]$. The negative resistance in them arises by lag alternate current from the alternate voltage Figure 1.

The dynamic negatrons have been used for filtering, frequency multiplexing and other microwave applications [3].

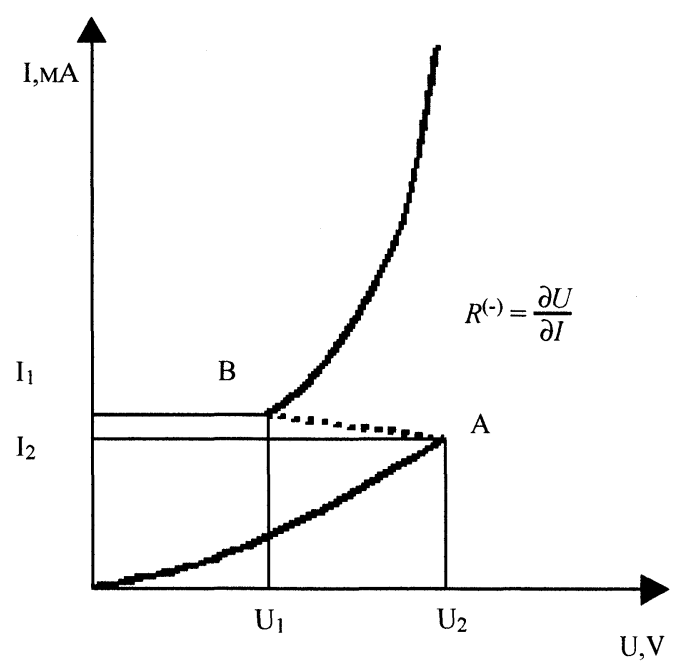

FIGURE 1a Static characteristics of S-type negatron.

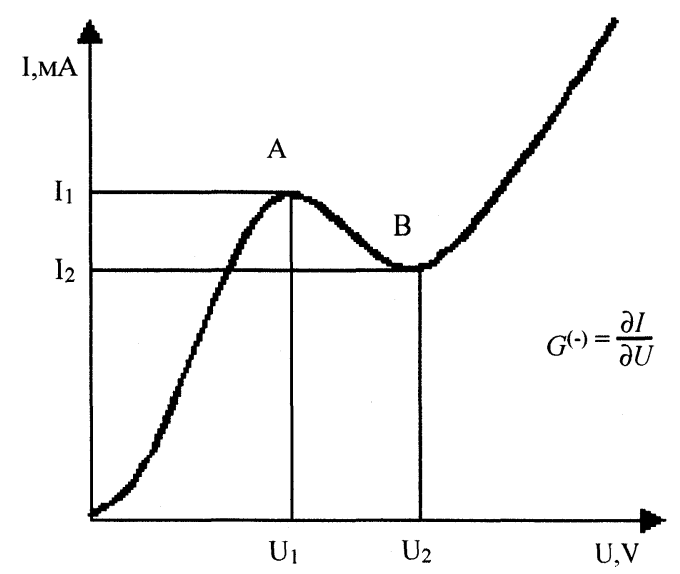

FIGURE 1b Static characteristics of N-type negatron. 


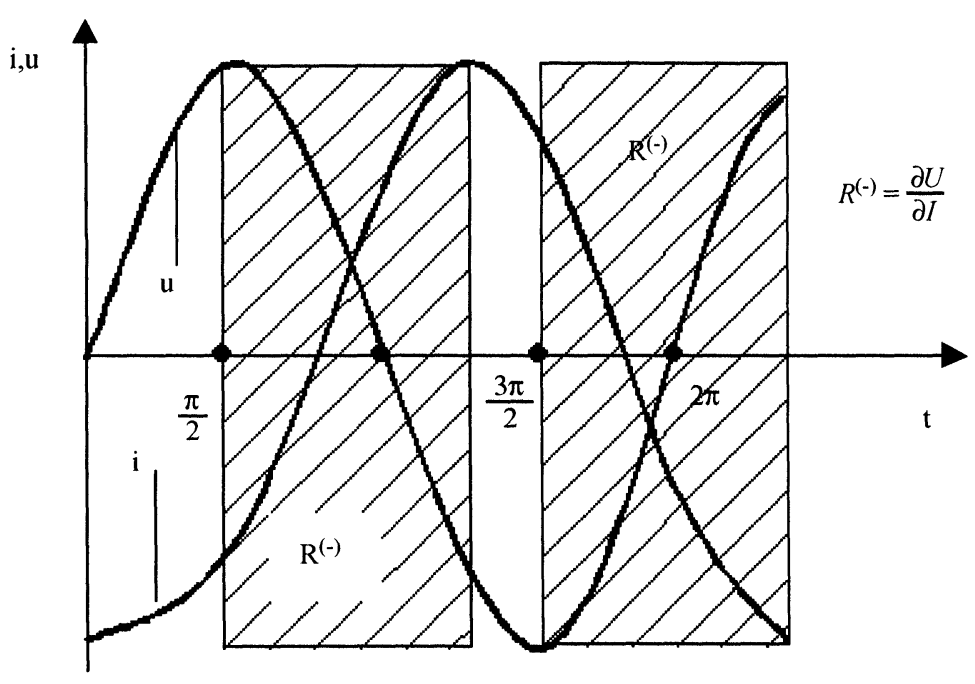

FIGURE 1c Time dependence of alternate current and voltage in dynamic negatron.

\section{NON-LINEAR MODEL}

A non-linear model of dynamic transistor negatrons has been developed on the basis of charge-model equations [3]. As shown in the previous works, the task of simulation of transit-time and injected processes in transistor structures is reduced practically to the simulation of accumulation and spreading of space charges, thus the simulation is based on the use of charge model. The influence of the space-injected charge of current-carriers on the opening and closing time of emitter junction has been taken into account in the model. The proposed non-linear model allows describing the stationary state of the negatron.

In the large-signal mode the charge carriers, which are injected from the emitter to the base, are accumulated in the base near emitter junction and create the volume charge $Q$. This volume charge discourages the carriers' injection from emitter. As the result, the emitter junction is closed rather early than in small-signal mode. The injected volume charge increases proportionally to the applied voltage and may be calculated through the diffusion capacity $C_{e d}$. Figure 2 


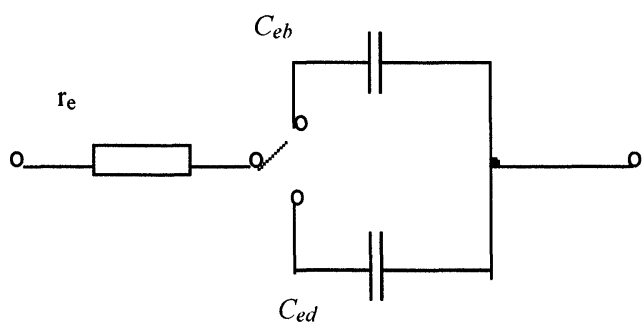

FIGURE 2 Where $C_{e b}$-barrier capacitance of emitter junction; $C_{e d}$-diffusion capacitance of emitter junction.

shows the equivalent circuit of the emitter junction in non-linear mode.

The switching of capacitances from $C_{e d}$ to $C_{e b}$ take place, when the emitter junction is closed.

If impulse of the injected emitter current is set as:

$$
i_{e}=I_{0}+I_{m} \sin \omega t,
$$

and the current of a diffusion capacity is equal to

$$
i_{c e}=\frac{d Q}{d t} .
$$

The diffusion capacity is a constant of proportionality between applied voltage and appropriate space charge $Q$ :

$$
C_{d e}=\frac{Q}{U}=\frac{1}{r_{e} \omega_{T}}
$$

where $U=\left(I_{0}+I_{m} \sin \omega_{T}\right) r_{e}$ - voltage on the emitter - base junction, $\omega_{T}$ - limiting frequency, which is the determined time of carriers for current transferring in base.

Then by substituting values of $Q$, in (1) we would get

$$
i_{c e}=\frac{d\left(I_{0}+I_{m} \sin \omega t\right) r_{e}\left(1 / r_{e} \omega_{T}\right)}{d t}
$$

and after manipulation:

$$
i_{c e}=I_{m} \frac{\omega}{\omega_{T}} \cos \omega t
$$


Condition of the closing of transistor's emitter junction will be the equality of the sum of active currents and capacity components of the junction:

$$
i_{e}+i_{c e}=0
$$

or after substitution of currents' values:

$$
I_{0}+I_{m} \sin \omega t_{1}+I_{m} \frac{\omega}{\omega_{T}} \cos \omega t_{1}=0 .
$$

After introduction of a dimensionless factor $Y=I_{0} / I_{m}$ the Eq. (2) takes the aspect:

$$
Y+\sin \omega t_{1}+\frac{\omega}{\omega_{T}} \cos \omega t_{1}=0 .
$$

By solving Eq. (3) for $\omega t_{1}$, we shall determine the angle of closing of the emitter junction $\varphi_{1}=\omega t_{1}$. Cosine component in Eq. (2) describes the charge's current of the diffuse capacity of the emitter junction. Time of opening of emitter junction $t_{2}$ and angle of opening $\psi_{2}=\omega t_{2}$ are find under condition when cumulative charge equals to zero, which flows back through the barrier capacity in the recharge process:

$$
\int_{t_{1}}^{t_{2}}\left(I_{0}-I_{m} \sin \omega t\right) d t=0
$$

As the corollary, input current of the transistor in large signal mode considerably differs from small-signal mode, and has a form of short impulse.

The input current of the non-linear mode is shown in Figure 3.

The voltage on the emitter could be circumscribed by a set of equations:

$$
\begin{gathered}
U_{e}=E^{\prime}-\frac{1}{4 E^{\prime} C_{e 0}^{2} \omega^{2}}\left(I_{0} \omega t+I_{m} \cos \omega t-I_{m}^{2}\right), \text { at } \omega t_{1}<\omega t<\omega t_{2} \\
U_{e}=E^{\prime}-\text { at } \omega t_{2}<\omega t<2 \pi+\omega t_{1} .
\end{gathered}
$$

The full equivalent resistance of emitter junction on frequency of the first harmonics is calculated as the ratio of complex amplitude of 


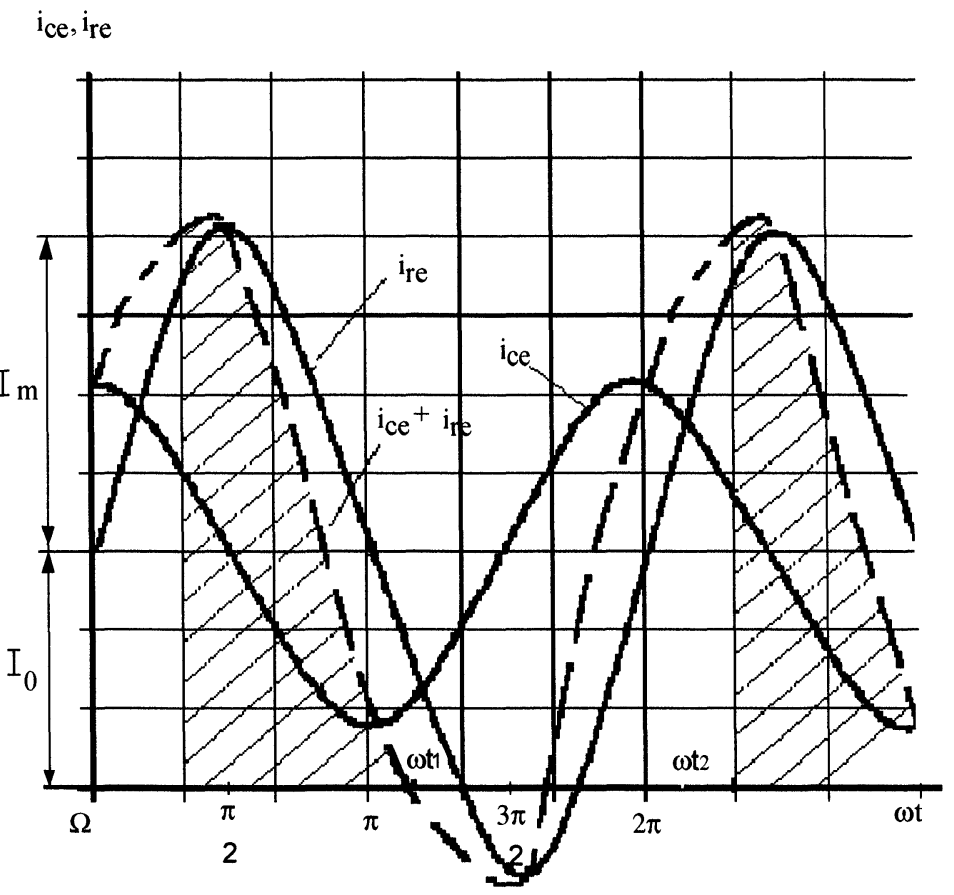

FIGURE 3

voltage on emitter junction to complex amplitude of emitter's current:

$$
Z_{e}=r_{e}+i x_{e}, \quad r_{e}=\frac{I_{0}}{4 \omega^{2} C_{e 0}^{2} E^{\prime}} F_{1}, \quad x_{e}=\frac{-I_{0}}{4 \omega^{2} C_{e 0}^{2} E^{\prime}} F_{2},
$$

where $F_{1}, F_{2}$-auxiliary factors, which are from the equations for $U_{21}$, $U_{22}$, appears in function $Y$.

In view of the entered improvements the charge equation is solved. We search solutions as periodic function. Thus it is possible to describe the composite equations, which appears in the function of time, Fourier series with a limited amount of terms. After substituting them in the charge equation it breaks up to a system of algebraic equations, which are solved by method of iteration.

The circumscribed theoretical model differs from known one, because it describes dynamic differential parameters of transistor's emitter junction. However, for full exposition of work of the transistor it is necessary to simulate the processes too, which occur in the field of 
base. The known models take into account current's transferring-time of carriers in the base, but they do not take into account the phase shift, which is between the current and voltage in the base and its dependence on amplitude of the signal. The proposed model, which takes into account the dependence of an equivalent resistance of base upon magnitude of a space charge, which is concerned with amplitude of the signal.

The complex resistance of base in view of modulation of conductivity is equal to:

$$
\begin{aligned}
Z_{b} & =\frac{K T}{q I_{E}} \frac{2}{b+1}\left[1+\frac{(1 / 2) h^{2} W^{2}(\log Q-1)}{1+(1 / 2) h^{2} W^{2}}\right]= \\
& =r_{b}\left\{1+\frac{(1 / 2)\left[\left(1+j \omega \tau_{h}\right) W^{2} /\left(D_{a} \tau_{h}\right)\right](\log Q-1)}{1+\left(1 /\left(2\left(1+j \omega \tau_{h}\right) W^{2} /\left(D_{a} \tau_{h}\right)\right)\right)}\right\}
\end{aligned}
$$

where

$\tau_{h}$-life-time of the non-ground carriers at a high level of injection; $D_{a}$-ambipolar coefficient of diffusion;

$\mu_{n}, \mu_{p}$-electron's and hole's mobility accordingly;

$Q$-charge of non-ground carriers, which are accumulated in base of the transistor.

Neglecting the magnitude $i \omega \tau_{h}$, as $j \omega \tau_{h} \ll 1$, we would get:

$$
Z_{b} \approx r_{b}\left[1+\frac{j \omega\left(L_{b 1} / r_{b}\right)}{1+j \omega\left(L_{b 1} / R_{1}\right)}\right]
$$

where,

$$
\begin{aligned}
& L_{b}=r_{b}\left(W^{2} / 2 D_{a}\right)(\log Q-1), \\
& R_{1}=R_{b}-r_{b}=r_{b}(\log Q-1), \quad \frac{L_{b 1}}{R_{1}}=\frac{W^{2}}{2 D_{a}} .
\end{aligned}
$$

Thus, the impedance of base of the transistor in view of modulation of conductivity can be presented as a circuit, which consists of a resistor $R_{1}$ and inductance $L_{1}$, magnitude of which depends on the current, which flows back through them. The obtained equations have physical significance. In the Eq. (4) $\log Q$ is proportional to longitudinal sequential voltage in active area of the base, which is 
defined by the equation:

$$
\log Q=\frac{q U_{b}}{K_{T}}
$$

Then the Eq. (4) with reference to (5) could be presented as

$$
L_{1}=\left(\frac{K T}{q I_{E}} \frac{2}{b+1}\right)\left(\frac{q U_{b}}{K_{T}}\right)\left(\frac{W^{2}}{K T}\right)=\frac{W^{2}}{2 D_{p}} \frac{U_{b}}{I_{E}}
$$

Or with the help of dimensionless factor:

$$
U_{b}=\frac{K T}{q}\left(\frac{1}{y}\right)
$$

where $D_{p}$-hole's coefficient of diffusion in base.

The complete equivalent circuit of transistor negatron, intends to expose a non-linear mode of negatron. The equivalent circuit designated as non-linear, maps its dependence of parameters from voltages, which are applied, and from currents, which flow back in the transistor. The equivalent circuit also takes into account the known processes, which arise at high level of injection: Vebster, Kirk, Erli and modulation of conductivity of basic effects Figure 4.

The inductance of the base in the circuit also takes into account external inductance of the feed-back circuit. With the help of obtained equivalent circuit by submitted values of currents and voltages, the factors of a matrix of transmission $[A]$, scattering $[S]$, conductivity $[Y]$, resistance $[Z]$ could be calculated. Equivalent circuit allows to calculate the $A T$ impedance matrix and its characteristics, when the currents and voltages of transistor junctions change Figures 5, 6.

The proposed non-linear model allows to describe the stationary state of negatron. The non-linear equations, which define currentvoltage dependencies, can be described by multiple Taylor power series, where the components, which include small-signal response, can be distinguished. Therefore the negatron can be described as a nonlinear system, consisting of linear and non-linear circuits.

The expressions for currents $i_{1}, i_{2}$, describe linear circuits through the convolution of pulse-periodical function (Volterra series) for the 


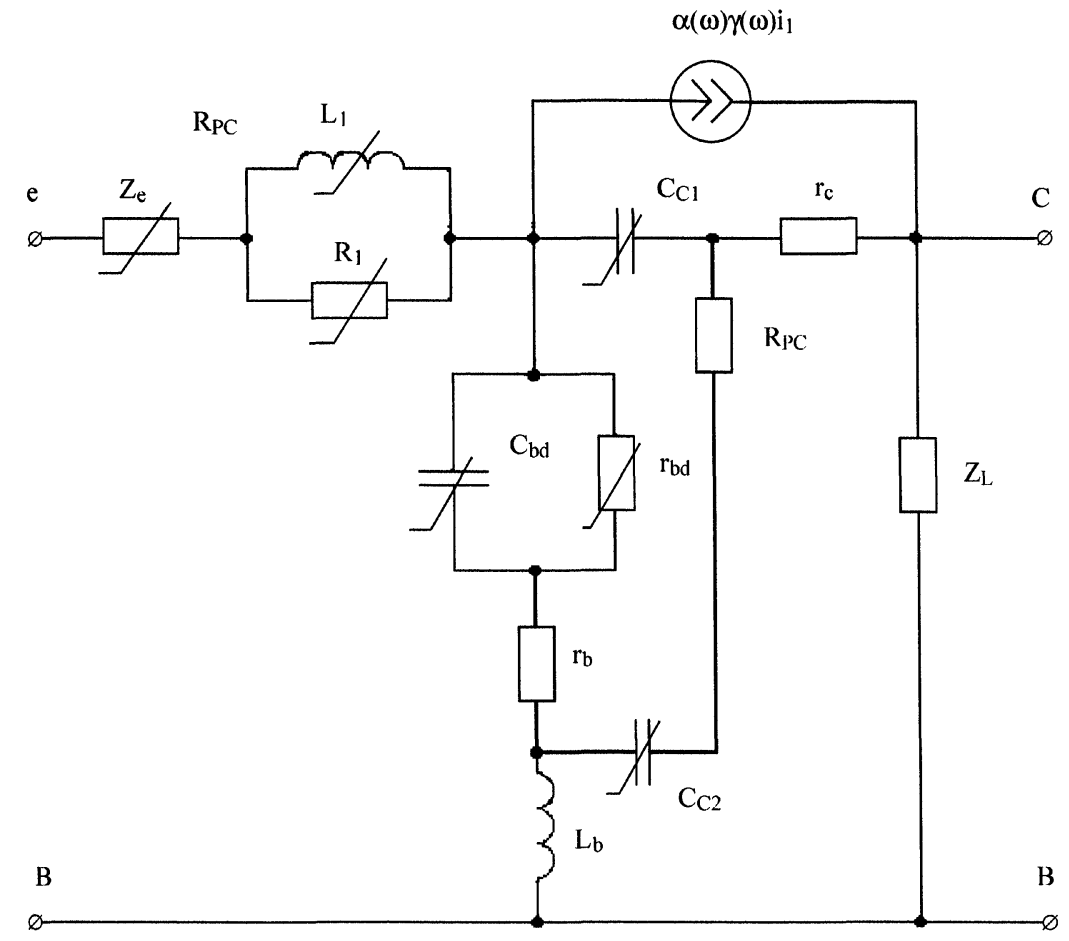

FIGURE 4 Equivalent circuit of the transistor negatron assigned to describe nonlinear mode.

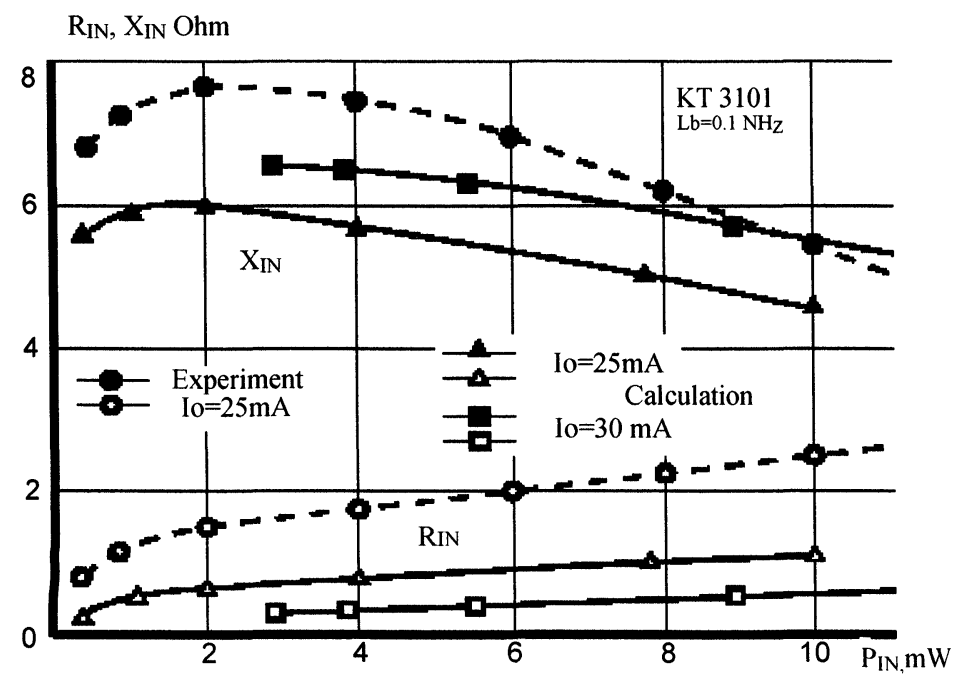

FIGURE 5 Components of impedance versus input power for different emitter currents characteristics. 


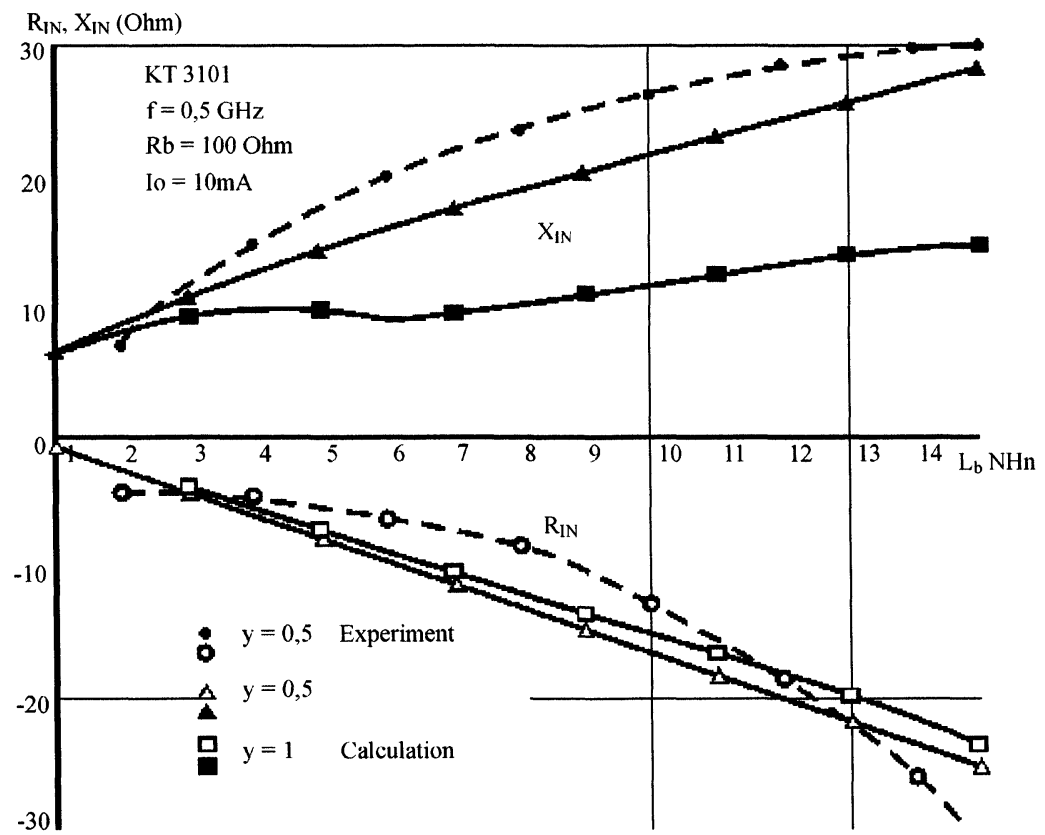

FIGURE 6 Impedance components versus feed-back inductance characteristics.

stationary mode

$$
\begin{aligned}
i_{1}= & \int_{0}^{\tau} a_{11}(\tau) \cdot u_{1}(t-\tau) d \tau+\int_{0}^{\tau} a_{12}(\tau) \cdot u_{2}(t-\tau) d \tau+ \\
& +i_{G e}(t)+\frac{d}{d t}\left[q_{e}(t)+\tau_{11} i_{G h}(t)\right]-\frac{\alpha_{I} \cdot i_{G h}(t)}{1+p \tau_{12}} \\
i_{2}= & \int_{0}^{\tau} a_{22}(\tau) \cdot u_{2}(t-\tau) d \tau+\int_{0}^{\tau} a_{21}(\tau) \cdot u_{1}(t-\tau) d \tau+ \\
& +i_{G h}(t)+\frac{d}{d t}\left[q_{h}(t)+\tau_{22} i_{G h}(t)\right]-\frac{\alpha_{N} \cdot i_{G e}(t)}{1+p \tau_{21}}
\end{aligned}
$$

where,

$$
\begin{gathered}
\alpha_{N}=\alpha_{N}\left(U_{e}, U_{\hat{e}}\right), \quad T+\frac{2 \pi}{w}, \quad a_{11}(t)=\frac{1}{T} \sum_{l=-L}^{L} Y_{11}^{L}(j w l) e^{j l w t} \\
a_{12}(t)=a_{21}(t)=\frac{1}{T} \sum_{l=-L}^{L} Y_{12}^{L}(j w l) e^{j l w t}, \quad a_{22}(t)=\frac{1}{T} \sum_{l=-L}^{L} Y_{22}^{L}(j w l) e^{j l w t}
\end{gathered}
$$


$Y_{p, q}$-elements of conductivity matrix of the transistor's linear circuit; $\square$-period of oscillation in system;

$i_{G e}, i_{G}-$ currents of emitter and collector junctions respectively;

$q_{e}, q$-charges of barrier capacitance of emitter and collector junctions;

$u_{1}, u_{2}-$ momentary input and output voltages;

$a_{N}, a_{l}-$ direct and reverse current gains;

$\tau_{p, q}$-time-constant in Hamilton's rmodel;

$p$-Laplas operator.

If the frequency's spectrum of input excitation is limited, then the matrix equation for four-port input excitation and response written by expressions

$$
\left[U_{c}\right]=\left[Y_{\Sigma}\right]^{-1}\left[I_{c}\right]
$$

where:

$$
\left[Y_{\Sigma}\right]=\left[\begin{array}{l}
Y_{11} Y_{12} \\
Y_{21} Y_{22}
\end{array}\right]
$$

- the square matrixes which responded to system of $Y$ parameters;

$$
\left[I_{c}\right]=\left[d I_{1}, d I_{2}\right]^{T}=\left[i_{-L_{1}}, \ldots, i_{01}, \ldots, i_{L_{1}}, i_{-L_{2}}, \ldots, i_{02}, \ldots, i_{L_{2}}\right]^{T}
$$

- the input current matrix vector, which describes the current's change in emitter and collector circuits on the combined frequency;

$$
\left[U_{c}\right]=\left[d U_{1}, d U_{2}\right]^{T}=\left[d U_{e}, d U_{k}\right]=\left[u_{-L_{1}}, \ldots, u_{L_{1}}, u_{-L_{2}}, \ldots, u_{L_{2}}\right]^{T}
$$

- the output voltage matrix vector, which describes the voltage in emitter and collector circuits on the combined frequency;

$$
\left[Y_{p q}^{L}\right]=\operatorname{diag}\left(Y_{-L p q}, \ldots, Y_{0 p q}, \ldots, Y_{L p q}\right)
$$

- the diagonal matrix, which include the linear part of circuit's conductance in main diagonal;

$\left[G_{e}\right],\left[G_{k}\right]$-the convolution form of the differential active conduction matrix, which characterise current-voltage junctions; 
$\left[C_{e}\right],\left[C_{k}\right]-$ the convolution form of the differential junctions capacitance matrix;

$$
[\Omega]=\operatorname{diag}\left(-L w+\Omega_{1}, \ldots, \Omega, \ldots, L w+\Omega\right),
$$

- the diagonal matrix of combination frequencies;

$[T]$ - the diagonal lag matrix;

$\left[G_{\alpha e}\right],\left[G_{\alpha \hat{e}}\right]$-conductance matrixes, which are the Fourier series coefficients as the functions product in time domain.

$$
p, q=1,2 ; \quad p+q=3 .
$$

The matrix expressions (6) defines the output change for amplifying and oscillating non-linear mode of dynamic negatrons.

Calculation and experimental measurement of input resistance of the investigated negatron circuits have shown that, at a defined relation of the load in a feed-back circuit, both currents and voltages of an active component feed of negatron resistance can accept a negative value, with exceeding loss of resistance. In this case, the negatron will work as the generator of sine wave oscillations. The developed models against known, permit us to carry out the calculation of oscillator parameters based on dynamic transistor negatrons. It has allowed the development of new circuits for oscillating devices and the

\begin{tabular}{|c|c|c|c|c|}
\hline $\begin{array}{l}\text { Type of } \\
\text { the } \\
\text { transistor }\end{array}$ & $\begin{array}{c}\text { Range of } \\
\text { frequencies, } \\
\text { GHz }\end{array}$ & $\begin{array}{c}\text { Broadband of } \\
\text { a frequency } \\
\text { modification, } \\
\text { MHz/\% }\end{array}$ & $\begin{array}{c}\text { Out-put power, } \\
M W\end{array}$ & $\begin{array}{c}\text { Efficiency, } \\
\%\end{array}$ \\
\hline $\begin{array}{l}\text { Oscillator } \\
\text { on } \\
\text { KT911B }\end{array}$ & $0,8 \div 1,6$ & $\begin{array}{c}800 \mathrm{MHz} \\
\text { or } \\
67 \%\end{array}$ & 200 & 6,7 \\
\hline GT313B & $0,3 \div 0,8$ & $\begin{array}{c}500 \mathrm{MHz} \\
\text { or } \\
98 \%\end{array}$ & 20 & 5 \\
\hline КТ913A & $0,6 \div 1,4$ & $\begin{array}{c}800 \mathrm{MHz} \\
\text { or } \\
67 \%\end{array}$ & 280 & 7 \\
\hline $\begin{array}{l}\text { Analog } \\
\text { (US) }\end{array}$ & 0,8 & $\begin{array}{c}240 \mathrm{MHz} \\
\text { or } \\
0,5 \%\end{array}$ & 250 & 5 \\
\hline $\begin{array}{l}\text { Analog } \\
\text { (Japan) }\end{array}$ & $0,98 \div 1,1$ & $\begin{array}{c}120 \mathrm{MHz} \\
\text { or } \\
11,5 \%\end{array}$ & 250 & 2,5 \\
\hline
\end{tabular}

TABLE I Comparative table for the parameters of investigated and known oscillators 


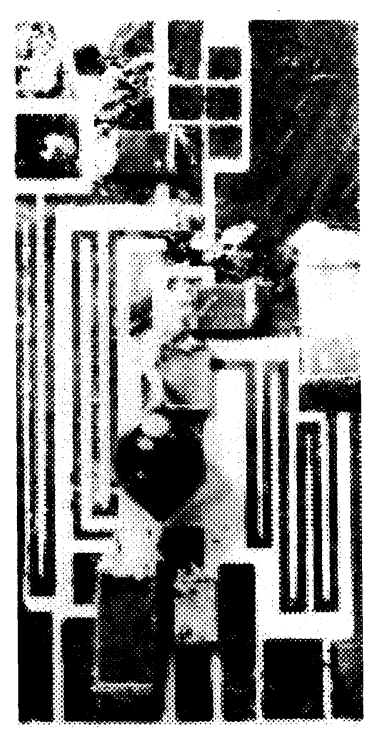

FIGURE 7

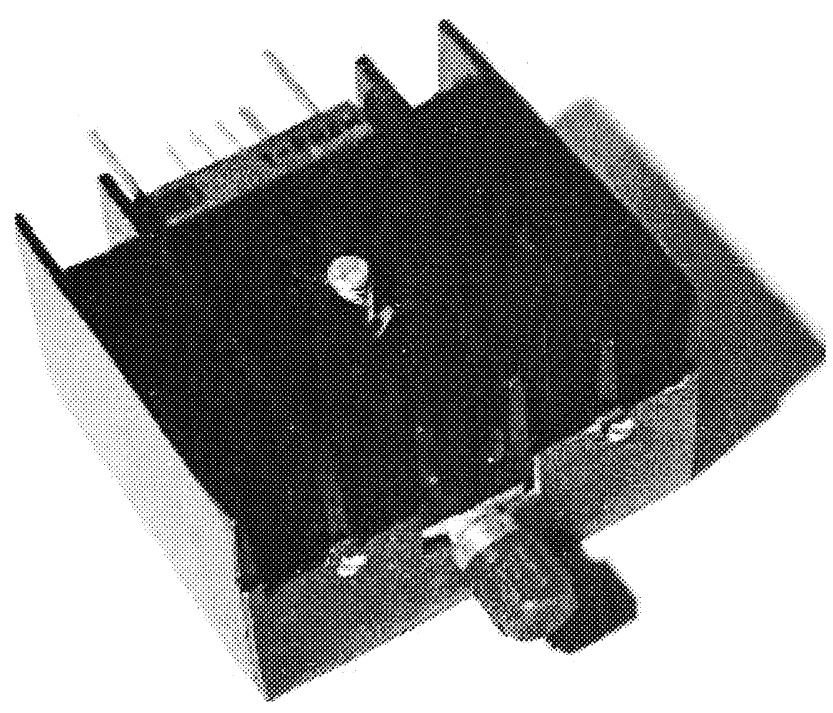

FIGURE 8

improvement of known circuit's performance. The parameters of the developed and investigated oscillators [3] are shown in Table I. The parameters of investigated transistors are shown in Table II. 
TABLE II Parameters of investigated oscillators

\begin{tabular}{lccccc}
\hline $\begin{array}{l}\text { Type of the } \\
\text { transistor }\end{array}$ & $\begin{array}{c}\text { Number of } \\
\text { transistors }\end{array}$ & $\begin{array}{c}\text { Frequency } \\
\text { GHz }\end{array}$ & $\begin{array}{l}\text { Pout } \\
\text { mW }\end{array}$ & $\begin{array}{c}\text { Kn } \\
\text { DB }\end{array}$ & $\begin{array}{c}\text { Error of } \\
\text { simulation \% }\end{array}$ \\
\hline $2 \square 391 \square-2$ & 2 & $2 \div 5$ & 40 & $2 \div 4,5$ & 15 \\
$2 \square 3101 \square-2$ & 2 & $1 \div 3$ & 20 & $4,2 \div 4,5$ & 18 \\
$2 \square 3114 \square-6$ & 2 & $1,5 \div 4,5$ & 60 & $1 \div 2$ & 12 \\
$2 \square 3115 \square-2$ & 1 & $2 \div 5$ & 10 & $2 \div 6$ & 12 \\
$2 \square 3124 \square-2$ & 3 & $4 \div 6$ & 15 & $2 \div 4$ & 22 \\
$2 \square 3134 \square-2$ & 3 (without corpus) & $0,5 \div 1,5$ & 15 & $2 \div 4$ & 15 \\
\hline
\end{tabular}

Experimental samples of negatronic oscillators are shown on Figures 7, 8.

The non-linear model is developed on the basis of the non-linear charge model. Equivalent circuit and Volterra series are used for calculation of parameters of dynamic negatrons. Expressions were obtained, which give the linear relation between current and voltage charges and allow to calculate the transferring characteristics. Experimental oscillators are described, that confirms the theoretical predictions.

\section{CONCLUSIONS}

1. The principles of monolithic microwave dynamic transistor negatrons are reduced that unlike known ones take into account the influence of the large signal, currents and voltages of a feed on dynamic differential parameters of negatrons.

2. With the help of developed models the analytical relations are obtained. Dynamic differential parameters of transistor negatrons have been investigate in a non-linear condition, which have enabled to receive new scientific outcomes-dynamic, current and load characteristics of negatrons, which were used for improving engineering and operating performances, and also construction of the new negatron devices.

3. The methods of theoretical and experimental research of non-linear transistor negatrons and devices on their basis, that unlike known ones allow to carry out researches of dynamic differential parameters of negatrons in a non-linear mode, that has enabled 
to check up adequacy of the proposed models and analytical relations.

4. On the basis of proposed models techniques of calculation of devices parameters for control systems have been developed based on non-linear transistor negatrons-tuneable oscillators.

\section{References}

[1] Filinyuk, N. $\square$., Garyinov, S. $\square$., Seryejnov, $\square$. N. and Stepanova, L. N., Negatronics. Novosibirsk.: Nauka, 1995, p. 320.

[2] Chang, C. and Itoh, T., Microwave Active Bandpass Filters Using Negative Resistance Circuits. IEEE MTT-S, 38(12), Dec., 1990, 1879-1884.

[3] Molchanov, P. $\square$. (1998). Basics of Nonlinear Theory of Transistor Negatrons. Monograph. Vinnitsa: "Universum-Vinnitsa", p. 207.

[4] Molchanov, P. A., A Nonlinear Dynamic Transistor Negatron Theory. Proceedings of NDES'99, Ronne, Denmark, July, 1999, pp. 95-99. 

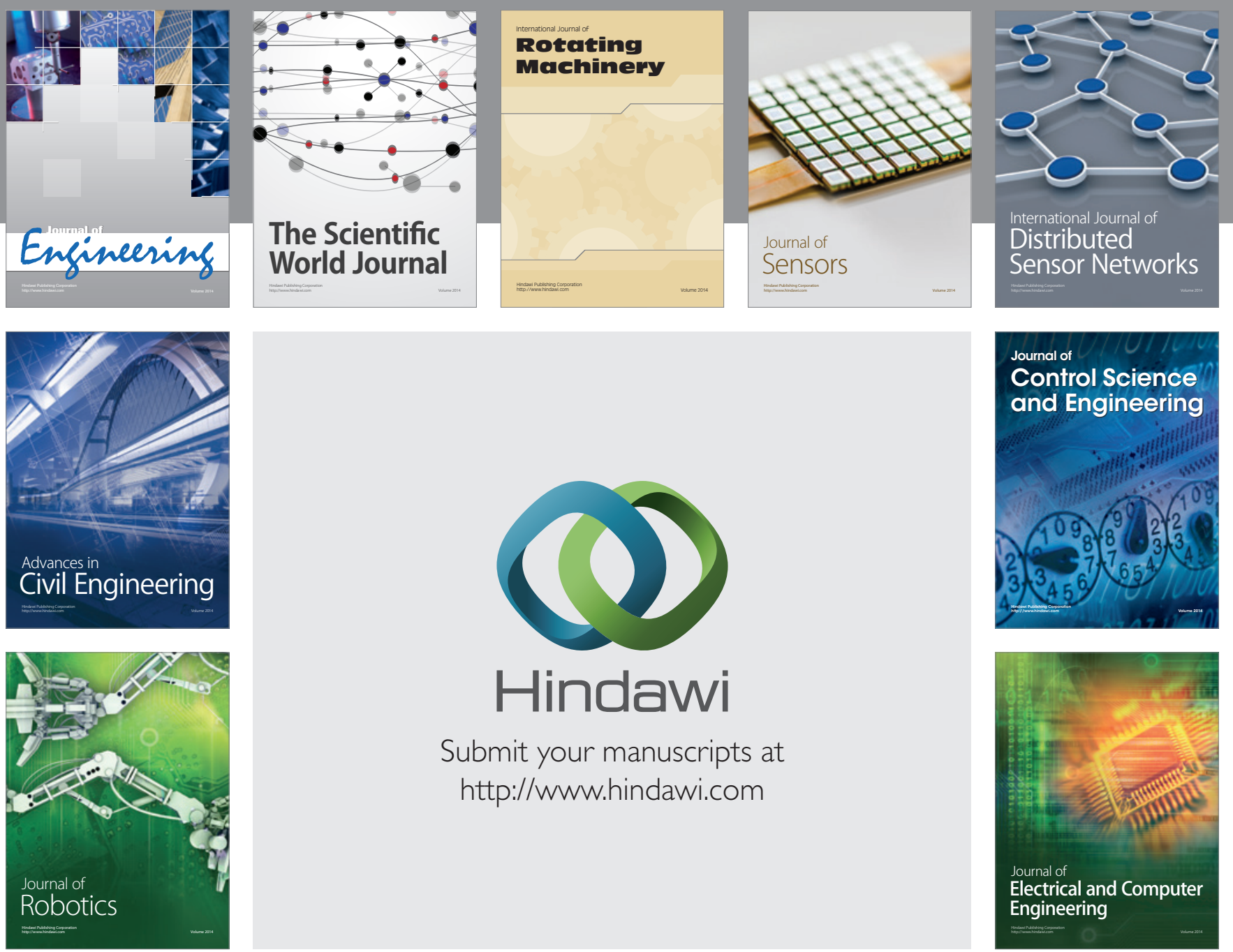

Submit your manuscripts at

http://www.hindawi.com
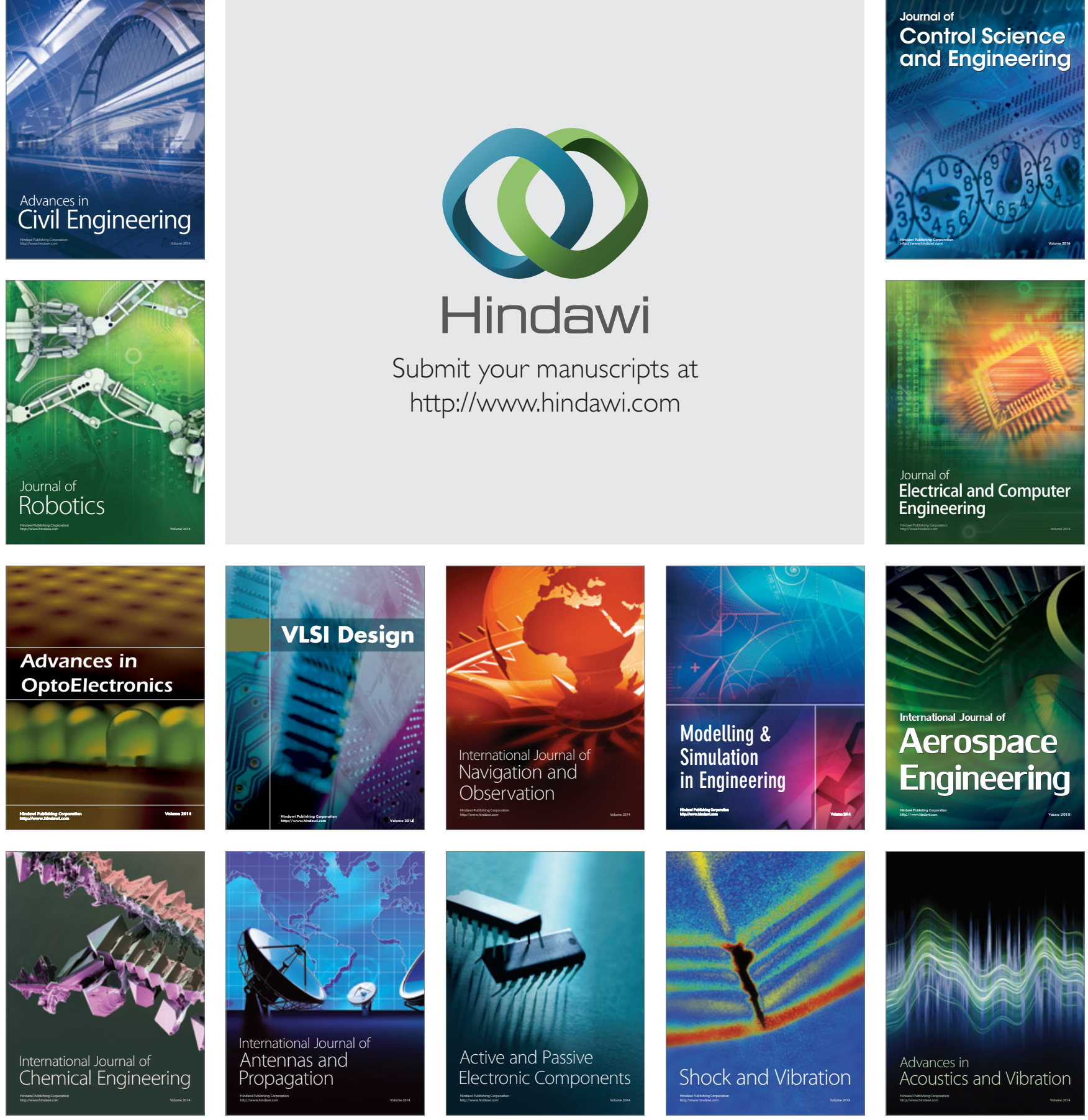